\title{
Simultaneous beam steering and shaping using a single-interface optofluidic component
}

\author{
Daniel Sauter $\odot$,* Merit Sieben, Pengpeng Zhao $\odot$, and Hans Zappe $\odot$ \\ University of Freiburg, Department of Microsystems Engineering-IMTEK, Gisela and \\ Erwin Sick Chair of Micro-Optics, Freiburg, Germany
}

\begin{abstract}
An optofluidic component actuated by electrowetting-on-dielectrics capable of simultaneously deflecting and shaping a beam in two dimensions, using a single liquid-liquid interface, is presented. The device features 32 individually addressable electrodes through which the interface is shaped using an open-loop control method to generate arbitrary surfaces. During steering-only operation, the resulting liquid prism can be rotated continuously with a repeatability of $\pm 0.071 \mathrm{deg}$ for individual positions. By choosing an appropriate surface shape, for example, it is possible to form a cylindrical lens, expanding the beam in only one axis, or to dynamically tune the scanned beam width. The presented results provide insights into the possibilities resulting from more complex surface control of optofluidic devices. (C) The Authors. Published by SPIE under a Creative Commons Attribution 4.0 International License. Distribution or reproduction of this work in whole or in part requires full attribution of the original publication, including its DOI. [DOI: 10.1117/1.JOM.1.4.044002]
\end{abstract}

Keywords: electrowetting; liquid prism; cylindrical lens; beam steering; beam shaping; optofluidics.

Paper 21022 received Aug. 5, 2021; accepted for publication Oct. 12, 2021; published online Oct. 29, 2021.

\section{Introduction}

Optical devices for beam steering and shaping traditionally consist of mechanical components, such as galvanometric mirrors, ${ }^{1}$ microelectromechanical system (MEMS) mirrors, ${ }^{2}$ rotating Risley prisms, ${ }^{3}$ or piezo actuators. ${ }^{4}$ However, these systems are often prone to failure due to mechanically moving parts, are expensive to manufacture, and have a high energy consumption. To overcome these drawbacks, impressive progress has been made in developing innovative alternative technologies, including electro-optic ${ }^{5}$ and acousto-optic systems, ${ }^{6}$ decentered prisms and lenses, ${ }^{7}$ and liquid-crystal-based optical systems. ${ }^{8}$ Another emerging technology for a wide range of tunable optical systems is optofluidics, using electrowetting-on-dielectrics (EWOD) as an actuation method. EWOD allows the manipulation of the shape of a liquid interface by applying an electric field and has been proven to be an attractive technology for tunable lenses and prisms, offering a large range of tunable focal lengths and steering angles, ${ }^{9,10}$ fast response times, ${ }^{11,12}$ low power consumption, ${ }^{12}$ and a high level of mechanical robustness. ${ }^{13}$ Due to its benefits, EWOD has a wide range of applications, including laser scanning systems, ${ }^{13}$ holographic displays, ${ }^{14}$ optical coherence tomography, ${ }^{15}$ as well as microscopy, ${ }^{16,17}$ optical switches, ${ }^{18}$ and solar concentrators. ${ }^{19,20}$

We present here an electrowetting-actuated optofluidic device with a single liquid interface that enables simultaneous beam steering and shaping with high resolution. The component combines a 360-deg rotatable and tiltable optofluidic prism, used for high resolution beam steering, with a new technique to incorporate variable curvature in two orthogonal axes. Creation of these complex shapes is realized by precise contact angle control achieved through a significantly increased electrode density in comparison to similar components. ${ }^{21-23}$ Thus, for example, a cylindrical lens of adjustable radius can be generated and rotated around the optical axis by $360 \mathrm{deg}$. Other systems are either comprised of multiple elements ${ }^{24,25}$ or provide very limited scan angles. ${ }^{15,22,26}$ Compared with these systems, the approach presented here delivers a large scan range of $\pm 5.1 \mathrm{deg}$ while only one device with a single interface is needed, offering

*Address all correspondence to Daniel Sauter, Daniel.Sauter@imtek.uni-freiburg.de 
significant advantages in terms of functionality and versatility. This fact combined with the ability of two-dimensional (2D) beam shaping will open up new possibilities in various application areas, such as automotive illumination systems. ${ }^{27,28}$

\section{Device and Experimental Setup}

The main component of the system is the cylindrical optofluidic device whose technological basis was established in the previous work. ${ }^{13}$ Two immiscible liquids of different refractive index are stacked in a glass tube which has a thin polymeric foil attached to its inner wall. The resulting liquid-liquid interface acts as a refractive surface used to manipulate light. Segmented electrodes are embedded in the foil that enables control of the surface shape through EWOD-actuation. This section describes the structure of the device and the experimental setup, and Sec. 3 explains the control approach and how the calculation of actuation voltages is carried out.

Figure 1(a) shows a schematic diagram of the device and its main components. Indicated in light blue and yellow are the aqueous and oil-based liquids that are encapsulated in the glass cylinder by two glass substrates. By applying different voltages to the electrode segments embedded in the foil, the liquid interface may be shaped as, for example, a tilted plane. The device configuration here differs from the lens-configuration used in earlier work ${ }^{29}$ in that the aqueous and oil phases are interchanged, so the glass substrate incorporating the ground electrode is located on the side of the light source. This arrangement allows for a beam path that is completely unobstructed by the ground electrode, even at high beam deflection angles.

The tube has an inner diameter of $5 \mathrm{~mm}$ and a length of $10 \mathrm{~mm}$ with additional $0.5 \mathrm{~mm}$ glass substrate thickness on each side. The active liquid is a blend of commercial components OHGL and OHZB; and the surrounding oil phase is Laser Liquid 433 (all available from Cargille Laboratories, catalog numbers 19580, 19581, 20108). Their kinematic viscosities are $\nu_{\mathrm{OHGL}}=679 \mathrm{cSt}, \nu_{\mathrm{OHZB}}=9 \mathrm{cSt}$, and $\nu_{433}=5 \mathrm{cSt}$ with densities of $\rho_{\mathrm{OHGL}}=1.254 \mathrm{~g} \mathrm{~cm}^{-3}$,

(a)

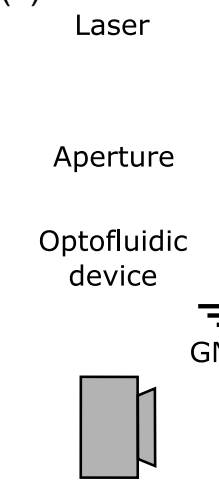

USB-microscope

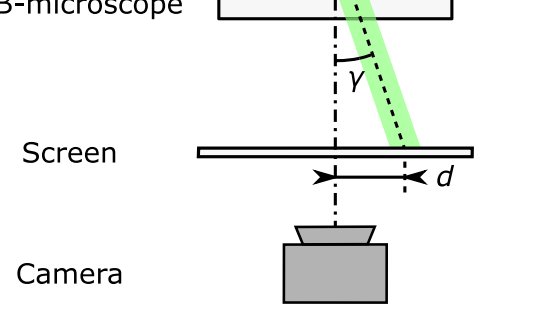

(b)

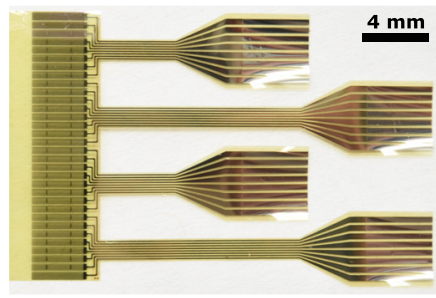

Foil with

electrodes

Aqueous

phase

Liquid

interface

Oil phase (c)

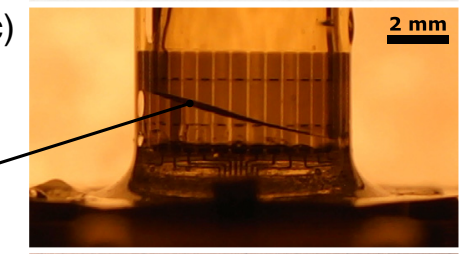

(d)

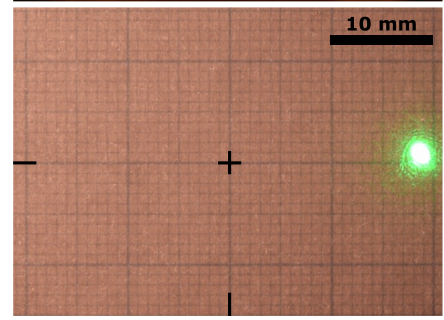

Fig. 1 (a) Schematic drawing of the optofluidic device and the experimental setup used to characterize it. (b) Polymeric foil prior to assembly. The electrode segments used to control the liquid interface are visible on the left, dark lines are conductive platinum paths leading from the electrodes to FFC connectors on the right. (c) Side view of the assembled optofluidic device with an applied target surface tilt angle of $\alpha=15$ deg. (d) Image of screen showing the beam deflection for the applied surface tilt. The black cross marks the optical axis and the laser is adjusted to hit this spot when the device is not in the beam path. Camera settings were adjusted to make grid visible. 
$\rho_{\text {OHZB }}=2.498 \mathrm{~g} \mathrm{~cm}^{-3}$, and $\rho_{433}=1.905 \mathrm{~g} \mathrm{~cm}^{-3}$. To eliminate gravity effects, the ratio of OHGL and OHZB is chosen such that the density of the resulting blend matches that of Laser Liquid 433. Their refractive indices are $n_{\mathrm{OHGL}+\mathrm{OHZB}}=1.499$ and $n_{433}=1.293$ at $589.3 \mathrm{~nm}$ and $25^{\circ} \mathrm{C}$.

Figure 1(b) shows a photograph of the polymeric foil. It features an electrode area composed of 32 individually addressable sections. Polyimide serves as the base material of the foil; and the transparent electrodes are made of indium tin oxide. Each of the electrode segments is contacted via a conductive platinum path of which eight are bundled into a flexible wire leading out of the housing. The wire interface is designed to directly fit a commercial flat flex cable (FFC) connector mounted on a custom circuit board. To ensure a large tuning range, the foil is coated with the hydrophobic fluoropolymer Cytop.

Standard planar microstructuring techniques are used to fabricate the foil. These enable the implementation of small features and consequently a high electrode density. After fabrication, the foil is rolled up and attached to the inner sidewall of the glass housing, as shown in Fig. 1(c). This transformation from a 2D structure into a three-dimensional device through assembly is the foundation that allows for great design freedom. A detailed description of the fabrication and assembly process is given in Ref. 13 .

In addition to the device itself, electronic hardware is needed to generate the actuation voltages for the electrode segments. For this purpose, a digital-to-analog converter (DAC) is used in conjunction with a high voltage amplifier. The 32 channel DAC (EVAL-AD5372, Analog Devices) is controlled via a MATLAB script and delivers 32 individually tunable voltages. In the case of DC-operation, these voltages are directly fed into the amplifier (40-ch $\mathrm{HV}$ amplifier, OKO Tech, Netherlands) and amplified by a fixed factor, leading to an output range of up to $400 \mathrm{~V}$. However, as DC-operation can lead to charge trapping that decreases the tuning range, AC-voltages may be used instead. Then, an additional custom multiplier circuit is introduced after the DAC that multiplies a $1 \mathrm{kHz}$ sine wave to the signals prior to amplification, leading to a maximum actuation voltage of $141 \mathrm{~V}_{\mathrm{RMS}}$. The period of this sine wave is significantly smaller than the measured response time of $219 \mathrm{~ms},{ }^{30}$ ensuring a stable liquid interface during operation. ${ }^{31}$

To characterize the device and demonstrate its abilities, the experimental setup shown in Fig. 1(a) was used. All measurements presented in Secs. 4 and 5 were obtained with this system. It consists of a collimated laser source $(532 \mathrm{~nm}, 0.9 \mathrm{~mW}$, CPS532-C2, Thorlabs, Germany) that is adjusted such that it hits the center of a grid screen. The optofluidic device and a circular aperture with a diameter of $1 \mathrm{~mm}$, to assure a well-defined beam profile, are then placed into the beam path. Two cameras are used to observe the behavior of the device: a USB-microscope provides a side view of the device that gives qualitative information about the surface shape while another camera captures the laser spot on the screen. The camera aimed at the screen (Nikon D5300, AF-S Micro-NIKKOR $60 \mathrm{~mm} \mathrm{1:2.8G} \mathrm{ED)} \mathrm{is} \mathrm{operated} \mathrm{with} \mathrm{fixed} \mathrm{settings}$ (ISO 100, F20, 1/100 s) and connected to a frame grabber to allow automatic capturing and processing.

After the definition of a target surface shape for the liquid-liquid interface [for example, a plane with tilt angle $\alpha$ as shown in Figs. 1(a), 1(c) and 1(d)], the corresponding voltages for each electrode segment are calculated and applied. As a result, the overall liquid shape changes, the laser beam is refracted at the interface, which results in a deflection angle $\gamma$ from the optical axis and a deviation $d$ of the spot on the screen. All measurements are performed with a device-screen distance of $213 \mathrm{~mm}$.

\section{Interface Control}

Essential for the operation of this device is the liquid interface control, i.e., how a target surface is defined and the actuation voltages determined. The open-loop approach used here is based on a purely geometrical surface description combined with measured voltage/contact-angle curves. No simulation steps are required, keeping the method simple and computationally inexpensive. We first provide here a conceptual description of the method, generalized to arbitrary surfaces and electrode segments. Application of the method is discussed in more detail in Secs. 4 and 5. 
First, a desired surface profile is chosen and expressed as height $z$ in polar coordinates $r$ and $\varphi$. This can be any function differentiable in $r$, e.g., a planar, cylindrical, or toroidal surface. Evaluating the partial derivative of $z(r, \varphi)$ with respect to $r$ at the radius of the inner tube wall $r_{\mathrm{T}}$ yields the slope along its circumference. The required contact angle associated with a certain electrode $\theta_{i}$ is then obtained through trigonometry simply as

$$
\theta_{i}=\left.\arctan \left[\left(\frac{\partial}{\partial r} z(r, \varphi)\right)^{-1}\right]\right|_{r=r_{\mathrm{T}}, \varphi=\varphi_{i}}
$$

where $\varphi_{i}$ denotes the angular position of the $i$ 'th electrode in polar coordinates. The angular positions of a device with $m$ equally spaced electrodes are given as

$$
\varphi_{i}=(i-1) \frac{2 \pi}{m},
$$

where $i$ is the electrode index ranging from 1 to $m$.

Figure 2(a) shows an example of a target surface. The angular electrode positions for $m=16$ segments are indicated with blue dots. These blue dots are where Eq. (1) is evaluated to determine the contact angle associated with each electrode.

To consider contact angle saturation and account for possible deviations in material properties or fabrication dimensions, the conversion of contact angle to voltage is done based on measurements. For the fabricated foil, a voltage-contact angle curve is recorded as shown in Fig. 2(b). The data are obtained using a dedicated test structure on the same wafer as the foil and a contact angle goniometer (OCA15Pro, DataPhysics Instruments GmbH, Filderstadt, Germany). Multiple forward and backward actuation cycles are recorded and then averaged. The data are interpolated using third-order Hermite interpolation to obtain a continuous and smooth curve. Actuation voltages are then easily obtained by evaluating the inverse interpolation function at the desired contact angle. The result is an array of $m$ voltages, one for each electrode segment as shown in Fig. 2(c).
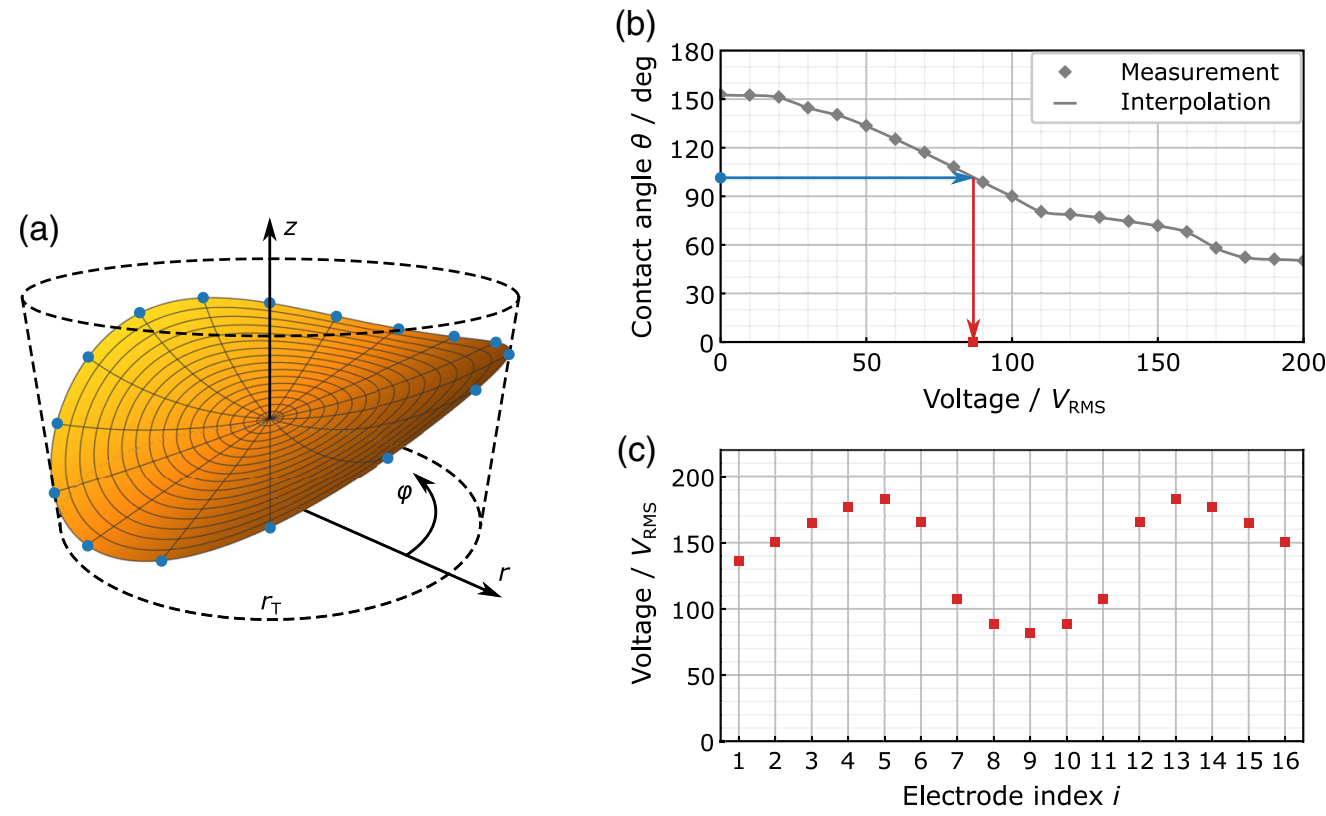

Fig. 2 (a) Mathematical description of a cylindrical surface with added tilt in the plane without curvature. Blue dots lie on the tube's inner radius $r_{\mathrm{T}}$ and indicate where the partial derivative is evaluated to calculate the contact angle. The example shows $m=16$ electrodes. (b) Measured contact angle curve used to calculate the voltages. Mean measurement values of forward and backward actuation are shown including their interpolation. (c) The calculated actuation voltages for each electrode for the provided example surface. 


\section{Beam Steering}

To characterize the fundamental scanning and positioning behavior of the device, it was first used solely as a rotatable prism. The previously described control method was used with the target liquid interface expressed as a tilted plane given as

$$
z(r, \varphi)=r \tan (\alpha) \cos (\varphi-\beta)
$$

where $\alpha$ is the desired surface tilt angle and $\beta$ is the surface rotation angle around the $z$ axis. This basic target surface description is equivalent to the truncated cylinder used in previous work and yields the same sinusoidal variation of contact angle along the circumference. ${ }^{13}$

Four example prism orientations are shown in Fig. 3(a) with their respective target values. The observed beam spots of the configurations were combined in Fig. 3(b). For a target tilt angle of $0 \mathrm{deg}(1)$, the calculated voltage is constant around the circumference and should ideally lead to a flat surface with a contact angle of $90 \mathrm{deg}$. However, a minor deviation of the laser beam from the optical axis occurred, indicating a slightly misaligned device or nonideal surface shape. For the actual prism configurations $(2,3,4)$, the expected beam deflections were observed with the tilt angle $\alpha$ determining the distance from the center and the rotation angle $\beta$ determining the angular position of the spot.

To determine the spatial resolution and precision of the scanner, a fixed tilt angle was applied to the device and the resulting liquid prism was rotated around the optical axis in discrete steps. For each step, an image of the screen was captured and the position of the laser spot was determined by calculating the intensity-weighted average position of pixels with an intensity greater than $50 \%$ of the sensor's maximum. Figure 4(a) shows measurement results for two different surface tilt angles and five actuation cycles. Average beam deflection angles of $\gamma=5.1 \mathrm{deg}$ and $\gamma=3.2 \mathrm{deg}$ were determined for the two target surfaces. Observed spot positions for each step are grouped closely together with an overall precision of $\pm 0.071 \mathrm{deg}$, calculated as the averaged standard deviations with respect to the mean. In comparison to the 16 -electrode-device, ${ }^{13}$ the precision was improved by a factor of more than 2, while the beam follows the ideal circular path much more closely and consistently for different tilt angles. As the used device materials and other technological factors have remained the same, we believe these improvements to be a consequence of the increased electrode density. On the one hand, more electrode segments enable a more accurate control of the liquid interface with less coarse discretization. On the other hand, the influence of deficiencies in individual electrodes on the whole system is smaller at higher electrode densities.

To discern whether the deviations from the ideal circular pattern stem from misalignment of the whole device or from manufacturing variations, the center position were examined. The measured center beam positions for a flat interface (square markers) show a small deviation from the optical axis. If this deviation was caused by a tilted device, this systematic error toward one side

(a)

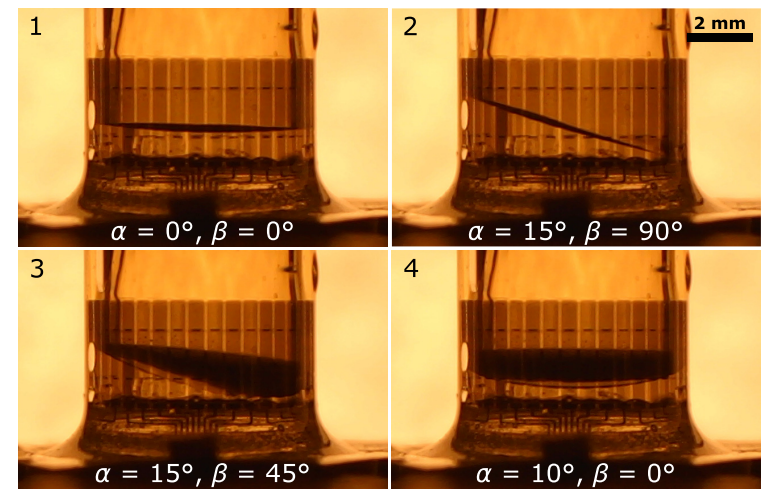

(b)

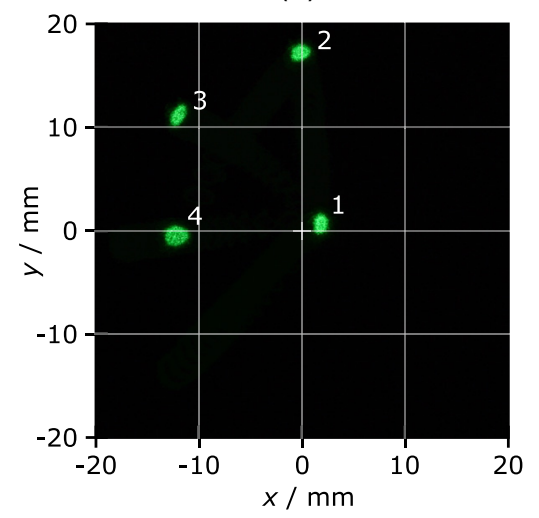

Fig. 3 (a) Side view of the meniscus shape for four different prism orientations as captured by the USB-microscope. Note that the microscope is oriented perpendicularly to the $y$ axis. (b) Observed beam spots on the screen for each configuration. 
(a)

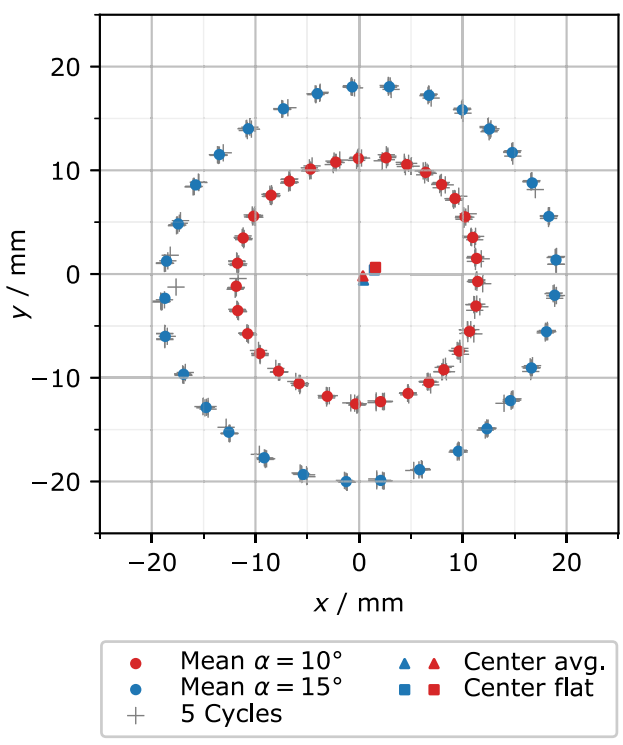

(b)

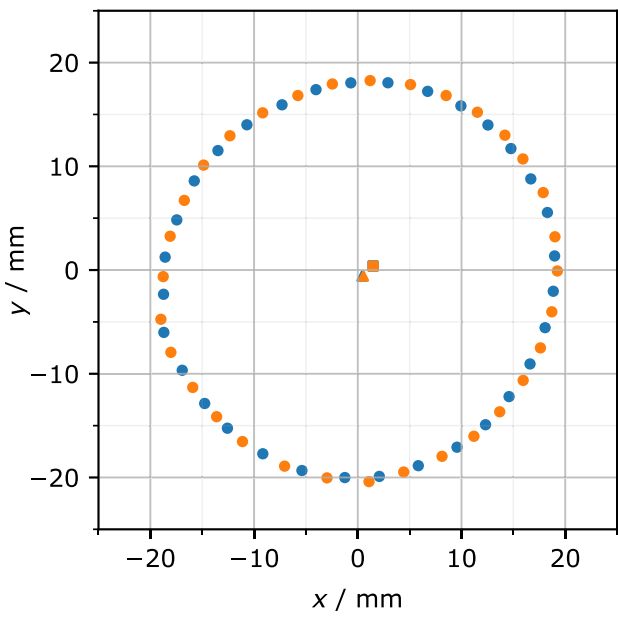

- $\alpha=15^{\circ}$ mid $\Delta \triangle$ Center avg

- $\alpha=15^{\circ}$ Center flat

Fig. 4 (a) Observed spot positions for target surface angles of 15 deg and 10 deg including the mean for each of the 32 discrete steps. Plot shows data of five actuation cycles. The triangle and box markers indicate the center obtained by averaging all data points and by measuring the laser spot when applying a flat surface, respectively. (b) The capability of reaching intermediate positions where the tilt axis lies between electrodes (orange). Only the mean positions of five cycles are shown. Of all shown data, the average standard deviation from the respective step mean is \pm 0.071 deg.

would also be present in the overall scanning pattern. However, the center position obtained by spatially averaging all positions (triangle markers) showed no such inclination-on the contrary, the pattern is centered remarkably well. This observation indicates that the deviations from the ideal scanning pattern are most probably caused by deficiencies from assembly or fabrication that are not accounted for during contact angle calibration.

The demonstrated maximum deflection angle of $\pm 5.1 \mathrm{deg}$ far exceeds those shown by other single-interface devices capable of combined tilt and focus control: the droplet-based devices ${ }^{22,26}$ are capable of $\pm 1 \mathrm{deg}$ and $\pm 2.13 \mathrm{deg}$, respectively, whereas the tubular device used in Ref. 15 has a range of $\pm 0.6 \mathrm{deg}$.

With the proposed control method, the surface rotation angles are not limited to $m$ discrete steps, as the parameter $\beta$ can be adjusted continuously. Figure 4(b) shows the scanning pattern for a surface tilt angle of $15 \mathrm{deg}$; the orange points were recorded with a target offset of $5.625 \mathrm{deg}$ in circumferential direction from the blue, placing them exactly in between two electrodes. The overall shape of the scanning pattern remained unchanged, and the precision was not influenced, indicated by a standard deviation of $\pm 0.074 \mathrm{deg}$ for the previous and $\pm 0.072 \mathrm{deg}$ for the offset pattern.

\section{Beam Shaping}

The ability to tune the contact angle in small sections along the circumference opens the opportunity to combine beam steering and beam shaping in a single liquid interface. For this purpose, the previously used surface description is extended with an additional term representing the curvature, namely

$$
z(r, \varphi)=r \tan (\alpha) \cos (\varphi-\beta)+\frac{\frac{r^{2} \cos ^{2}(\varphi)}{R_{\mathrm{x}}}+\frac{r^{2} \sin ^{2}(\varphi)}{R_{\mathrm{y}}}}{1+\sqrt{1-\frac{r^{2} \cos ^{2}(\varphi)}{R_{\mathrm{x}}^{2}}-\frac{r^{2} \sin ^{2}(\varphi)}{R_{\mathrm{y}}^{2}}}},
$$


(a)

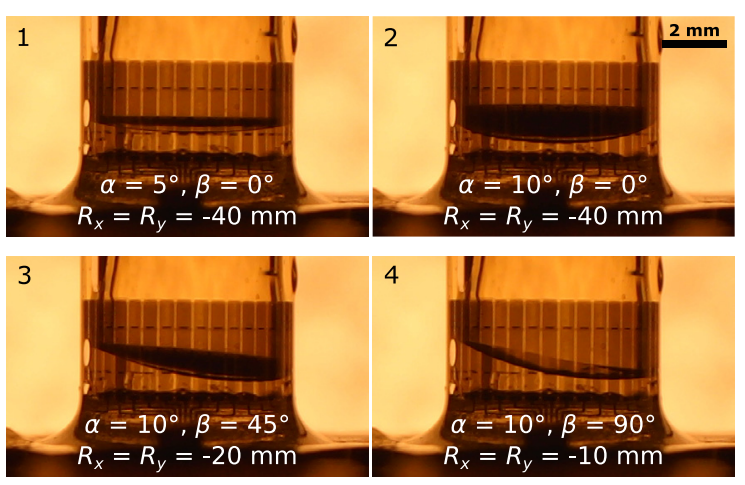

(b)

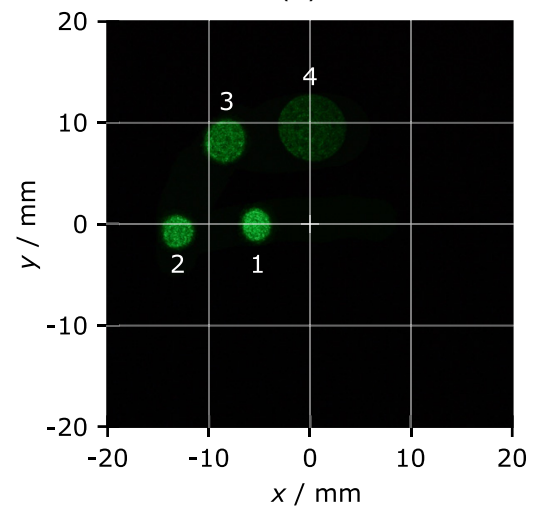

Fig. 5 Demonstration of spot size tuning with four different target configurations. (a) Meniscus side-view and (b) overlaid captured laser spots on the screen. 1 and 2 are configurations with a low curvature and thus smaller spot size. For configurations 3 and 4 , the curvature was increased. Note that the side-view microscope is oriented perpendicularly to the $y$ axis.

where the first term represents the tilted plane [Eq. (3)] and the second represents a double-plane symmetric curved surface. The description is derived from a biconic surface [Ref. 32, Eq. (5-78)], the conic constants of which were set to zero, and which was then transformed to polar coordinates. The resulting expression describes a double-plane symmetric surface with a circular profile whose radii of curvature $R_{x}$ and $R_{y}$ can be set independently for the two orthogonal axes.

A first demonstration of the utility of this concept was to control the circular spot size while steering the beam. Since the incoming laser beam is collimated, varying the spot size is easily achieved by changing the flat meniscus to a spherical surface. Different levels of defocus thus result in different circular spot sizes. A spherical surface is expressed using Eq. (4) by setting the radius in both axes to the same value. Figure 5 shows a set of example configurations, demonstrating the ability of varying the spot size with simultaneous tilt and rotation of the surface.

Figure 5(a)-4 clearly shows the intended surface shape of a tilted spherical lens. As shown in Fig. 5(b), the overall shape of the laser spots remained circular for all configurations. Configurations 1 and 2 were chosen to investigate the influence of an increased tilt angle while keeping the target curvature constant, but no change in size was observed. Next, the radius of curvature was decreased to $-20 \mathrm{~mm}$ and then to $-10 \mathrm{~mm}$, resulting in a further increase in spot size.

A more interesting application of the description given by Eq. (4) is that of an actual doubleplane symmetric shape, i.e., a surface whose radii in the two orthogonal planes are not equal. One special case is that of a cylindrical surface, where one plane has a curvature of zero. Thus, the resulting surface has optical power only in one direction; Fig. 6 shows multiple examples of such cylindrical configurations highlighting different aspects, such as the tuning of the line length, rotation, and applying simultaneous tilt.

Examples (a) and (b) in Fig. 6 show beam profiles for a purely cylindrical target surface. In both cases, the beam width in the $x$ direction was nearly unaffected while an elongation of the spot in the orthogonal direction occurred. The length of the resulting laser line was adjusted by varying the radius of curvature.

Subsequently, a tilt angle was added to the target description to determine whether it is possible to tilt the cylindrical lens surface in the same way as for the spherical lens. Three cases were chosen: tilt along the flat $x$ axis 6(d); tilt along the curved $y$ axis 6(e); and tilt along an arbitrary axis between the two 6(f). All three cases showed the desired behavior of simultaneous beam steering and shaping, although some irregularities were present. While some shift in the lateral position of the spot was noted, the angular orientation of the laser lines remained remarkably stable throughout all configurations. This observation shows that the angular orientation of the tilted plane and that of the curved surface can be set individually in Eq. (4) to vary the position and orientation of a laser line independently. 


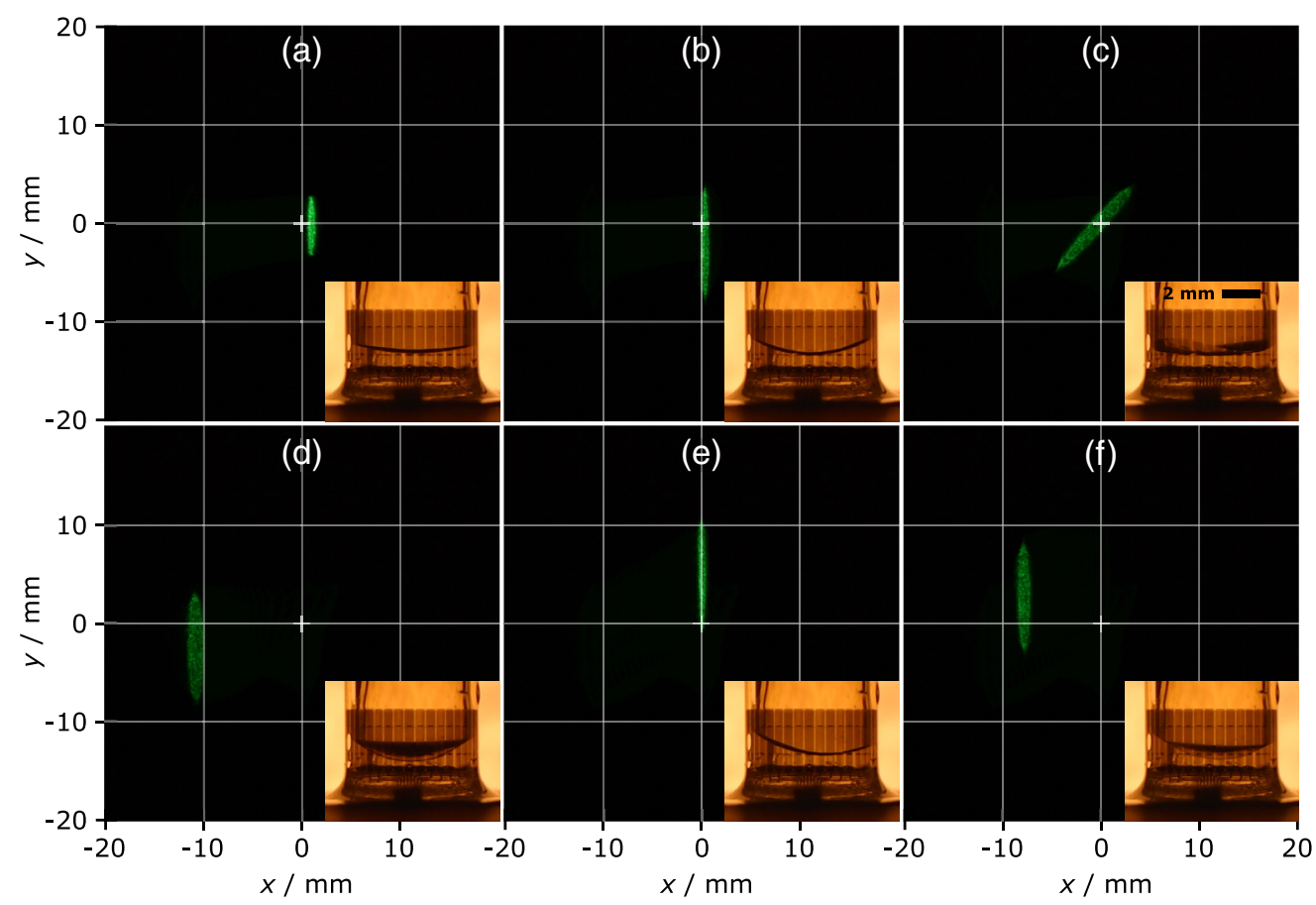

Fig. 6 Laser lines generated by shaping the liquid interface into a cylindrical lens shape. The radius in the $x$ direction $R_{x}$ was set to infinity for all configurations. (a) Radius in $y$ direction $R_{y}=-15 \mathrm{~mm}$, (b) $R_{y}=-5 \mathrm{~mm}$, (c) $R_{y}=-5 \mathrm{~mm}$ rotated by $45 \mathrm{deg}$, (d) $R_{y}=-5 \mathrm{~mm}$ with surface tilt of $\alpha=10$ deg along $x$ axis, (e) $R_{y}=-5 \mathrm{~mm}$ with surface tilt of $\alpha=10$ deg along $y$ axis, and (f) $R_{y}=-5 \mathrm{~mm}, \alpha=10 \mathrm{deg}$ with the tilt axis oriented at $\beta=45 \mathrm{deg}$.

The results of Fig. 6 show clearly that the basic principle of simultaneous beam steering and shaping functions as desired. Nevertheless, some nonideal aspects of the spot shape remain, particularly unwanted increase in line width. The high degree of surface control available using this fluidic concept can now be used to improve the beam shape: for example, manipulating the initially flat orthogonal axis can be used to compensate unwanted spreading of the laser line, with the goal of maintaining a high aspect ratio and sharp line width for increased tilt angles. Figure 7(a) shows recorded line profiles for a target surface with a radius of curvature of $-5 \mathrm{~mm}$ in the $y$ direction and no curvature in the $x$ direction at different tilt angles. The spreading in the transverse direction at higher tilt angles is clearly visible. For each of the tilt angles, the target

(a)

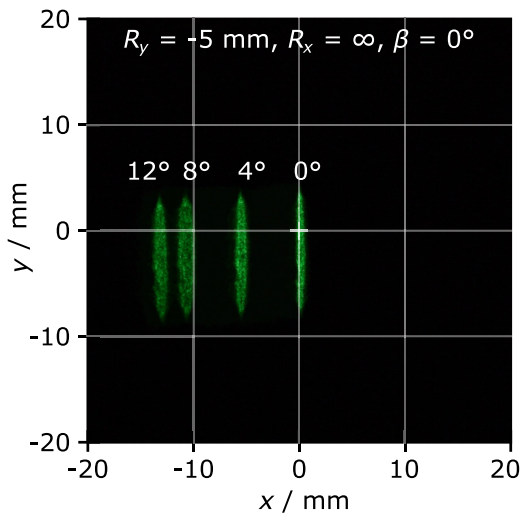

(b)

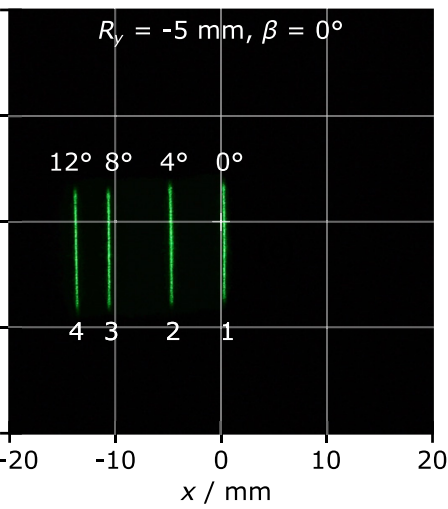

(c)

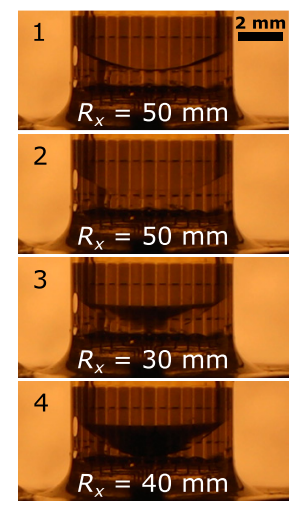

Fig. 7 (a) Laser lines at different tilt angles while keeping the curvature in $x$ direction zero, (b) same tilt angles but with applied curvature in the other direction to compensate for spreading, and (c) side-view of corresponding interfaces. 
curvature in $x$ direction was manually varied until the minimal line width was found. The resulting lines are shown in Fig. 7(b) with their respective values and observed surface shapes given in Fig. 7(c).

For all cases, applying a positive radius led to a significantly improved line shape. Line width after compensation was almost identical across all tested tilt angles, showing the effectiveness of the method. The curvature needed for compensation corresponded to the degree of the tilt-induced spreading present in the lines before, e.g., the line at 8 deg tilt required the largest curvature. The position of the spots was unaffected by the additional curvature.

In summary, the results prove that simultaneous beam steering and shaping using a single interface is feasible. Although simple in nature, the used open-loop control method provided a viable basis for defining complex surface shapes and compensating for irregularities. The device was capable of reshaping a circular laser beam independently in two axes which enabled the creation of elliptical profiles up to very high aspect ratios. Compared with liquid cylindrical lenses that are defined by the device geometry, ${ }^{33-35}$ the approach presented here, using only EWOD-actuation, offers a more flexible and attractive approach for lens tuning.

Nonetheless, there are factors that limit the performance of the device. Typical for EWODdevices are contact angle saturation and hysteresis. ${ }^{36}$ The former is addressed using measured contact angle curves while the influence of the latter is reduced to some degree by the applied AC voltage and could be reduced further by incorporating wetting feedback. ${ }^{37,38}$ Material inconsistencies, such as fluid density mismatch, local differences in wetting characteristics or simply manufacturing and assembly tolerances negatively impact the scanning accuracy but can be compensated by performing calibration. ${ }^{39}$ The scanning speed depends on the liquid properties that are identical to the ones used in the 16-electrode device. ${ }^{13}$ Recent effort to decrease the response time using shaped voltage input ${ }^{30}$ has shown promising results but is not implemented in the work presented here.

\section{Conclusion}

We have successfully demonstrated the application of an electrowetting-actuated optofluidic device to perform simultaneous beam steering and beam shaping. The device features a single liquid-liquid interface that is shaped via 32 individually addressable electrodes, allowing for a high degree of surface control. Generation of complex surfaces with independently adjustable tilt and curvature was achieved, enabling the liquid interface to not only act as a prism for beam deflection but to also dynamically reshape a beam.

The ability to simultaneously adjust the position and shape of a light spot in 2D opens up interesting new applications for EWOD optofluidic devices: nonmechanical adaptive illumination systems for automotive or display applications, laser line generation for triangulation, or particle measurements are all possible. Moreover, the existing control method proved to be an effective tool that can be further enhanced by integrating feedback about the beam's position and shape for calibration before or live correction during operation. Reconfigurable devices such as the one presented in this paper, with multiple integrated functions, no mechanical moving parts and good scalability, show the remarkable potential EWOD devices have.

\section{Acknowledgments}

This work was funded by the German Science Foundation (DFG) within the framework of the project optomechanical limits of tubular optofluidics (Project No. 411766042).

\section{References}

1. Z. Zhakypov, E. Golubovic, and A. Sabanovic, "Galvanometric optical laser beam steering system for microfactory application," in IECON 2013 - 39th Annu. Conf. IEEE Ind. Electron. Soc., IEEE, pp. 4138-4143 (2013).

2. X. Zhang et al., "Wide-angle structured light with a scanning MEMS mirror in liquid," Opt. Express 24(4), 3479-3487 (2016). 
3. A. Li et al., "Investigation of beam steering performances in rotation Risley-prism scanner," Opt. Express 24(12), 12840-12850 (2016).

4. W. Zhu et al., "Modeling and control of a two-axis fast steering mirror with piezoelectric stack actuators for laser beam tracking," Smart Mater. Struct. 24(7), 075014 (2015).

5. S. R. Davis et al., "Analog, non-mechanical beam-steerer with 80 degree field of regard," Proc. SPIE 6971, 69710G (2008).

6. D. Trypogeorgos et al., "Precise shaping of laser light by an acousto-optic deflector," Opt. Express 21(21), 24837-24846 (2013).

7. B. D. Duncan, "Wide-angle decentered lens beam steering for infrared countermeasures applications," Opt. Eng. 43(10), 2312 (2004).

8. Z. He et al., "Liquid crystal beam steering devices: principles, recent advances, and future developments," Crystals 9(6), 292 (2019).

9. M. Zohrabi, R. H. Cormack, and J. T. Gopinath, "Wide-angle nonmechanical beam steering using liquid lenses," Opt. Express 24(21), 23798-23809 (2016).

10. M. Zohrabi et al., "Lidar system with nonmechanical electrowetting-based wide-angle beam steering," Opt. Express 27(4), 4404-4415 (2019).

11. S. Kang, M. Duocastella, and C. B. Arnold, "Variable optical elements for fast focus control," Nat. Photonics 14(9), 533-542 (2020).

12. D. Kopp and H. Zappe, "Tubular focus-tunable fluidic lens based on structured polyimide foils," IEEE Photonics Technol. Lett. 28(5), 597-600 (2016).

13. D. Kopp, L. Lehmann, and H. Zappe, "Optofluidic laser scanner based on a rotating liquid prism," Appl. Opt. 55, 2136-2142 (2016).

14. C. Liu et al., "Electrowetting-actuated multifunctional optofluidic lens to improve the quality of computer-generated holography," Opt. Express 27(9), 12963-12975 (2019).

15. S. Kim et al., "Design and implementation of a low-cost, portable OCT system," Biomed. Opt. Express 9, 1232-1243 (2018).

16. O. D. Supekar et al., "Two-photon laser scanning microscopy with electrowetting-based prism scanning," Biomed. Opt. Express 8(12), 5412-5426 (2017).

17. L. Li et al., "Zoom microscope objective using electrowetting lenses," Opt. Express 24(3), 2931-2940 (2016).

18. M. Zohrabi et al., "High extinction ratio, low insertion loss, optical switch based on an electrowetting prism," Opt. Express 28(5), 5991-6001 (2020).

19. J. Cheng and C.-L. Chen, "Adaptive beam tracking and steering via electrowettingcontrolled liquid prism," Appl. Phys. Lett. 99(19), 191108 (2011).

20. J. Cheng, S. Park, and C.-L. Chen, "Optofluidic solar concentrators using electrowetting tracking: concept, design, and characterization," Solar Energy 89, 152-161 (2013).

21. I. Khan, S. Castelletto, and G. Rosengarten, "Two-axis electrowetting liquid lens for beam steering," Proc. SPIE 11486, 114860C (2020).

22. S. Seo et al., "Liquid microlens enabling tunable focus and tilt for resolution enhancement of 3d image," in 20th Int. Conf. Solid-State Sens. Actuators and Microsyst. Eurosens. XXXIII (TRANSDUCERS EUROSENSORS XXXIII), pp. 1592-1595 (2019).

23. M. B. Stokholm et al., "3D-imaging: a scanning light pattern projector," Appl. Opt. 55, 9074-9083 (2016).

24. J. Lee, J. Lee, and Y. H. Won, "Nonmechanical three-dimensional beam steering using electrowetting-based liquid lens and liquid prism," Opt. Express 27, 36757-36766 (2019).

25. C. Liu et al., "Multifunctional optofluidic lens with beam steering," Opt. Express 28(5), 7734-7745 (2020).

26. S. Berry et al., "Fluidic microoptics with adjustable focusing and beam steering for single cell optogenetics," Opt. Express 25, 16825-16839 (2017).

27. T. Asari et al., "Adaptive driving beam system with mems optical scanner for reconfigurable vehicle headlight," J. Opt. Microsyst. 1(01) (2021).

28. Y.-C. Fang et al., "A study of high-efficiency laser headlight design using gradient-index lens and liquid lens," Appl. Sci. 10(20), 7331 (2020).

29. P. Zhao, D. Sauter, and H. Zappe, "Tunable fluidic lens with a dynamic high-order aberration control," Appl. Opt. 60, 5302-5311 (2021). 
30. P. Zhao, Y. Li, and H. Zappe, "Accelerated electrowetting-based tunable fluidic lenses," Opt. Express 29, 15733-15746 (2021).

31. J. Park et al., "Critical AC frequency for stable operation of electrowetting-driven optofluidic devices with polymeric electrolyte solutions," J. Mech. Sci. Technol. 33, 1793-1797 (2019).

32. H. Gross, Handbook of Optical Systems: Vol. 1. Fundamentals of Technical Optics, Wiley-VCH, Weinheim (2005).

33. N. R. Smith et al., "Agile wide-angle beam steering with electrowetting microprisms," Opt. Express 14(14), 6557-6563 (2006).

34. E. Ozgur et al., "Parametric dog-bone-shaped tunable cylindrical fluidic lens," Appl. Opt. 60(16), 4755-4761 (2021).

35. H. Chen, N. Tabatabaei, and A. Amirfazli, "Liquid bridge as a tunable-focus cylindrical liquid lens," Appl. Phys. Lett. 110(4), 041608 (2017).

36. F. Mugele and J.-C. Baret, "Electrowetting: from basics to applications," J. Phys.: Condens. Matter 17, R705-R774 (2005).

37. F. Li and F. Mugele, "How to make sticky surfaces slippery: contact angle hysteresis in electrowetting with alternating voltage," Appl. Phys. Lett. 92(24), 244108 (2008).

38. B. de Boer et al., "Control of an electrowetting-based beam deflector," J. Appl. Phys. 107(6), 063101 (2010).

39. W. Y. Lim et al., "Calibration and characteristics of an electrowetting laser scanner," IEEE Sens. J. 20(7), 3496-3503 (2020).

Daniel Sauter received his BEng degree in mechatronics from Baden-Wuerttemberg Cooperative State University Stuttgart in 2015 and his MSc degree in embedded systems engineering from the University of Freiburg in 2020. Currently, he is a PhD candidate at the Gisela and Erwin Sick Chair of Micro-Optics. His research interests are focused on optofluidic devices and electrowetting.

Merit Sieben received her BSc and MSc degrees in microsystems engineering from the University of Freiburg in 2017 and 2020, respectively. Since 2020, she has been working as a $\mathrm{PhD}$ candidate at the Gisela and Erwin Sick Chair of Micro-Optics. Her research interests include the development of optofluidic tunable illumination systems whose beam profiles and radiance patterns can be digitally programmed.

Pengpeng Zhao received her MSc degree in optical engineering from the Harbin Institute of Technology in 2012. Same year, she joined the Gisela and Erwin Sick Chair of Micro-Optics as a $\mathrm{PhD}$ student, developing MEMS endoscopic components. Since 2017, she has been a postdoctoral researcher in the Gisela and Erwin Sick Chair of Micro-Optics, specializing on miniaturized optofluidic devices.

Hans Zappe is Gisela and Erwin Sick Professor of Micro-Optics at the University of Freiburg in Germany. He received his BSc and MSc degrees from MIT in 1983 and his PhD from the University of California, Berkeley, in 1989, all in electrical engineering. He worked at IBM (USA), the Fraunhofer Institute for Applied Solid State Physics (Germany), and the Centre Suisse d'Electronique et de Microtechnique (Switzerland), before joining the Department of Microsystems Engineering at the University of Freiburg in 2000. He is a fellow of SPIE, OSA, and IOP. 